\title{
3D RANS computations of open channel flows with a sharp bend
}

\author{
I. Kimura \& S. Takimoto \\ Hokkaido University, Sapporo, Japan \\ K. Blanckaert \\ École Polytechnique Fédérale de Lausanne, Lausanne, Switzerland \\ Y. Shimizu \\ Hokkaido University, Sapporo, Japan \\ T. Hosoda \\ Kyoto University, Kyoto, Japan
}

\begin{abstract}
This paper describes a 3D computation of an open channel flow with a sharp bend. The flow with a bend is characterized by generation of secondary current of the first kind due to the local unbalance of the centrifugal force and lateral pressure gradient. Since a nonlinear k- $\varepsilon$ model combined with a coarser computational grid is applied for the turbulence computation, the present method becomes much less expensive than LES approaches. The computation was performed under the same conditions of the laboratory test conducted by Blanckaert (2002). The present computation could capture qualitatively the main cell of the secondary current as well as a small cell called "outer-bank cell". The agreement of profiles of velocities and Reynolds stresses is also shown to be satisfactorily. Considering the computational efficiency, it is pointed out that the present RANS approach is a practical tool for prediction of open channel flows with a sharp bend.
\end{abstract}

\section{INTRODUCTION}

The turbulent flow structures in rivers at a curved part are characterized by the generation of the secondary current of the first kind caused by an unbalance of centrifugal force and a local pressure gradient. Since the secondary current is an important factor for sediment transport, the bed topography in rivers is closely affected by the secondary currents. It should be noted that a small vortex called an outer-bank cell is generated between the main cell of a secondary current and a side wall. Though scale of the outer-bank cell is much smaller than the secondary current of the first kind, the outer-bank cell may affect bank erosion because it appears at the vicinity of the bank. In order to predict the flow resistance as well as river morphology at a bend, the flow and the turbulent properties have to be clarified satisfactorily. However, due to the complicated flow properties including an outer-bank cell, precise prediction of the structure of the flow at river bends is difficult. In previous researches, it has been shown that a $3 \mathrm{D}$ computational method based on a non-linear RANS model is an effective tool for simulating open channel flows with a mild bend (Kimura et al., 2008). An advantage of RANS models compared with LES approaches is computational efficiency. However, the applicability of the RANS model to flows with a sharp bend has not been clarified yet. In case of sharp bends, more complicated flow patterns, such as rapid change of cross-sectional flow pattern and flow separation near an inner bank is generated. In this study, a non-linear RANS model is applied to the sharp curved open channel flows studied experimentally by Blanckaert (2002). 


\section{COMPUTATIONAL METHOD}

\subsection{Basic equations}

The Reynolds averaged 3D flow equations (1)-(4) with contravariant components of velocity vectors on a generalized curvilinear coordinate system are adopted as governing equations.

$$
\begin{aligned}
& \frac{1}{\sqrt{g}} \frac{\partial V^{\alpha} \sqrt{g}}{\partial \xi^{\alpha}}=0 \\
& \frac{\partial V^{i}}{\partial t}+\nabla_{j}\left[V^{i}\left(V^{j}-W^{j}\right)\right]+V^{i} \nabla_{j} W^{j}+V^{j} \nabla_{j} W^{i}=F^{i}-\frac{1}{\rho} g^{i j} \nabla_{j} p+\nabla_{j}\left[-\overline{v^{i} v^{j}}\right]+2 v \nabla_{j} e^{i j} \\
& \frac{\partial k}{\partial t}+\nabla_{j}\left[k\left(V^{j}-W^{j}\right)\right]+k \nabla_{j} W^{j}=-g_{i l} \overline{v^{l} v^{j}} \nabla_{j} V^{i}-\varepsilon+\nabla_{j}\left\{\left(\frac{D_{t}}{\sigma_{k}}+v\right) g^{i j} \nabla_{i} k\right\} \\
& \frac{\partial \varepsilon}{\partial t}+\nabla_{j}\left[\varepsilon\left(V^{j}-W^{j}\right)\right]+\varepsilon \nabla_{j} W^{j}=-C_{\varepsilon 1} \frac{\varepsilon}{k} g_{i l} \overline{v^{l} v^{j}} \nabla_{j} V^{i}-C_{\varepsilon 2} \frac{\varepsilon^{2}}{k}+\nabla_{j}\left\{\left(\frac{D_{t}}{\sigma_{k}}+v\right) g^{i j} \nabla_{i} \varepsilon\right\}
\end{aligned}
$$

where $\xi^{i}=$ generalized curvilinear coordinate, $t=$ time, $V^{i}=$ contravariant component of the velocity vector of flows, $W^{i}=$ contravariant component of the velocity vector of grid motion, $p=$ pressure, $v=$ molecular dynamic viscosity, $\rho=$ density of water, $k=$ turbulent energy, $\varepsilon=$ turbulent energy dissipation rate, $g_{i j}$ and $g^{i j}=$ covariant and contravariant component of metric tensor, $g=\operatorname{det}\left(g_{i j}\right)$ and $F^{i}=$ contravariant component of gravitational acceleration. $\nabla^{i}$ indicates a covariant differential, for instance,

$$
\nabla_{i} A^{k}=\frac{\partial A^{k}}{\partial \xi^{i}}+A^{j} \Gamma_{i j}{ }^{k}
$$

where $\Gamma_{\mathrm{ij}}{ }^{\mathrm{k}}=$ Christoffel symbol. The metric tensors and Christoffel symbol are calculated by

$$
g_{i j}=\frac{\partial x^{k}}{\partial \xi^{i}} \frac{\partial x^{l}}{\partial \xi^{j}} \delta_{k l}, g^{i j}=\frac{\partial \xi^{k}}{\partial x^{i}} \frac{\partial \xi^{l}}{\partial x^{j}} \delta_{k l}, g_{i j} g^{j k}=\delta_{i}^{k}, \Gamma_{i j}{ }^{k}=\left\{\begin{array}{c}
k \\
i j
\end{array}\right\}=\frac{1}{2} g^{k m}\left(\frac{\partial g_{j m}}{\partial \xi^{i}}+\frac{\partial g_{i m}}{\partial \xi^{j}}-\frac{\partial g_{i j}}{\partial \xi^{m}}\right)
$$

\subsection{Turbulence models}

We adopted two-equation RANS type model with the second order non-linear terms considering computational efficiency. The constitutive equation of the present model is:

$$
\begin{aligned}
& -\overline{v^{i i} v^{j j}}=D_{t} S^{i j}-\frac{2}{3} k \delta_{s}^{i} g^{s j}-\frac{k}{\varepsilon} D_{t}\left[\alpha_{1} Q_{1}+\alpha_{2} Q_{2}+\alpha_{3} Q_{3}\right], D_{t}=C_{\mu} \frac{k^{2}}{\varepsilon} \\
& Q_{1}=S^{i \alpha} g_{\alpha l} \Omega^{l j}+S^{j \beta} g_{\beta l} \Omega^{l i}, Q_{2}=S^{i \alpha} g_{\alpha l} S^{l j}-\frac{1}{3} S^{k \alpha} g_{\alpha m} S^{m \beta} g_{\beta k} \delta_{l}^{i} g^{l j}, \\
& Q_{3}=\Omega^{i \alpha} g_{\alpha l} \Omega^{l j}-\frac{1}{3} \Omega^{k \alpha} g_{\alpha m} \Omega^{m \beta} g_{\beta k} \delta_{l}^{i} g^{l j} \\
& S^{i j}=g^{j \alpha} \nabla_{\alpha} V^{i}+g^{i \alpha} \nabla_{\alpha} V^{j}, \Omega^{i j}=g^{j \alpha} \nabla_{\alpha} V^{i}-g^{i \alpha} \nabla_{\alpha} V^{j}
\end{aligned}
$$

The model coefficients should not be constants but functions of the strain parameter $\mathrm{S}$ and the rotation parameter $\Omega$ to satisfy realizability (e.g., Kimura \& Hosoda, 2003). In this study, the following coefficients proposed by Ali et al (2007) are employed.

$$
\begin{aligned}
& \alpha_{1}=-0.1325 f_{M}, \alpha_{2}=0.0675 f_{M}, \alpha_{3}=-0.0675 f_{M}, f_{M}=\left(1+m_{d s} S^{2}+m_{d \Omega} \Omega^{2}\right)^{-1} \\
& C_{\mu}=c_{\mu 0}\left(1+c_{n s} S^{2}+c_{n \Omega} \Omega^{2}\right) / D_{\mu}
\end{aligned}
$$




$$
\begin{aligned}
& D_{\mu}=1+c_{d s} S^{2}+c_{d \Omega} \Omega^{2}+c_{d s \Omega} S \Omega+c_{d s 1} S^{4}+c_{d \Omega 1} \Omega^{4}+c_{d S \Omega 1} S^{2} \Omega^{2} \\
& S=\frac{k}{\varepsilon} \sqrt{\frac{1}{2} S^{i \alpha} g_{\alpha j} S^{j \beta} g_{\beta i}}, \quad \Omega=\frac{k}{\varepsilon} \sqrt{\frac{1}{2} \Omega^{i \alpha} g_{\alpha j} \Omega^{j \beta} g_{\beta i}}
\end{aligned}
$$

The values of the model constants in equations (10)-(12) are listed in Table 1. The detailed process of tuning the model constants has been reported by Ali et al (2007).

Table 1. Model constants.

\begin{tabular}{rccccccc}
\hline $\mathrm{C}_{\beta 1}$ & & $\mathrm{C}_{\beta 2}$ & & $\mathrm{C}_{\beta 3}$ & $\mathrm{~m}_{\mathrm{dS}}$ & $\mathrm{m}_{\mathrm{d} \Omega}$ \\
\hline 0.4 & & 0 & & -0.13 & 0.01 & 0.003 \\
\hline \hline $\mathrm{c}_{\mathrm{nS}}$ & $\mathrm{c}_{\mathrm{n} \Omega}$ & $\mathrm{c}_{\mathrm{dS}}$ & $\mathrm{c}_{\mathrm{d} \Omega}$ & $\mathrm{c}_{\mathrm{dS} \Omega}$ & $\mathrm{c}_{\mathrm{dS} 1}$ & $\mathrm{c}_{\mathrm{d} \Omega 1}$ & $\mathrm{c}_{\mathrm{d} \Omega \Omega 1}$ \\
\hline 0.0028 & 0.007 & 0.0085 & 0.004 & -0.003 & 0.00005 & 0.00005 & 0.00025 \\
\hline
\end{tabular}

\section{COMPUTATIONAL CONDITIONS}

\subsection{Outline of the laboratory test by Blanckaert (2002)}

Blanckaert (2002) carried out a laboratory experiment using an experimental flume schematically shown in Figure 1. The curvature radius at the bend is $\mathrm{R}=1.7 \mathrm{~m}$, the width of the channel is $\mathrm{B}=1.3 \mathrm{~m}$, the average depth is $\mathrm{H}=0.159 \mathrm{~m}$. The channel is composed of 3 parts, i.e., a $9 \mathrm{~m}$ long straight inflow part, curved part with $193^{\circ}$ center angle and a $5 \mathrm{~m}$ long outlet channel. He performed two kinds of experiments: the experiment with a horizontal bed and the experiment with a movable bed. We employ the simple horizontal bed case (H89) as a testing data. The flow discharge of case $\mathrm{H} 89$ is $\mathrm{Q}=891 / \mathrm{s}$. The Reynolds number and Froude number with average depth and average velocity are 6840 and 0.34 , respectively. The bed is covered with sand of $d_{50}=2 \mathrm{~mm}$. The sand particles were fixed on the bed by a paint sprayed on it. The three-dimensional velocity profiles at section $15^{\circ}, 30^{\circ}, 60^{\circ}, 90^{\circ}, 120^{\circ}, 150^{\circ}$ and $180^{\circ}$ are measured using an acoustic Doppler velocity profiler. The acquisition time was $180(\mathrm{sec})$.

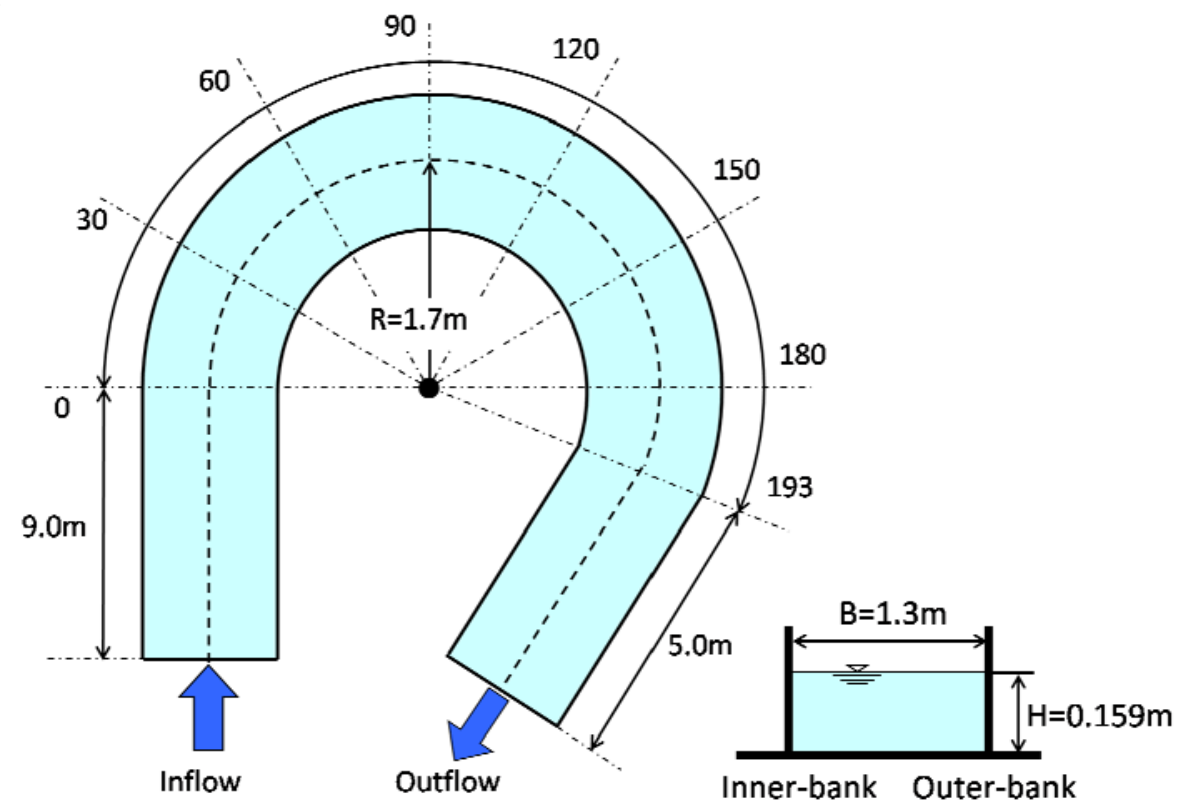

Figure 1. Schematic diagram of curved channel (Blanckaert(2002)).

\subsection{Computational domain and grid}

The horizontal view of the computational grid is shown in Figure 2. The grid is tuned through grid sensitivity analysis. The number of the grid cells in $\mathrm{x}, \mathrm{y}$ and $\mathrm{z}$ directions are 84,50 and 16, respectively. Each straight channel part at inlet and outlet is $1 \mathrm{~m}$ long. In order to obtain the inlet 
boundary conditions, a preliminary computation in a straight channel (length $=1.0 \mathrm{~m}$ ) was performed using cyclic boundary conditions at inlet and outlet boundaries. The cross-sectional flow and turbulence profiles of the preliminary computation are used as the inlet boundary conditions.

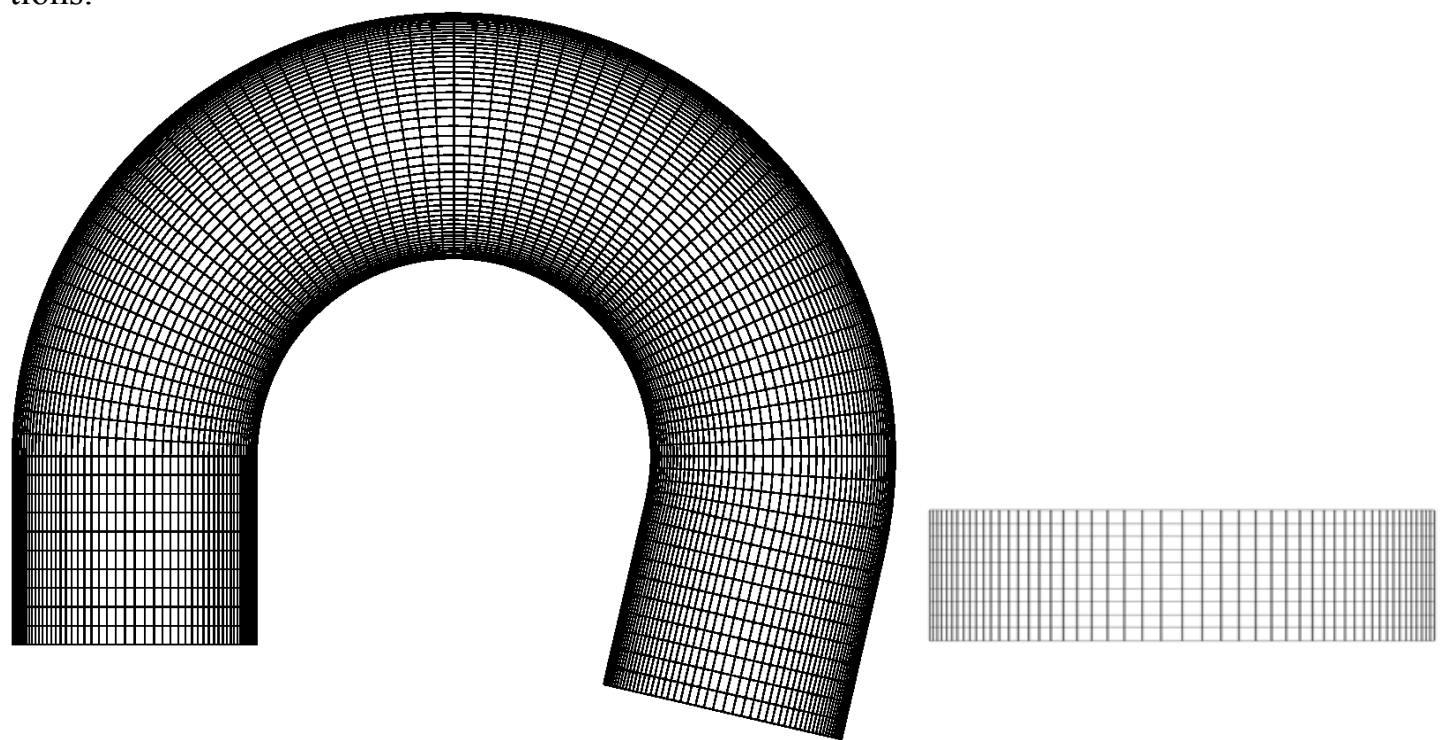

Figure 2. Computational grid in the horizontal and cross-sectional section.

\section{RESULTS AND DISCUSSIONS}

\subsection{Cross sectional flow patterns}

Figure 3 shows the comparison of cross-sectional velocity vectors at $30^{\circ}, 60^{\circ}, 90^{\circ}$ and $120^{\circ}$ by both the laboratory test and the present computations. Two kinds of recirculation cells can be seen at each section with the experimental result. The larger cell with the clockwise direction is the main circulation of the secondary current of the first kind and the smaller cell at the vicinity of the outer-bank with the anti-clockwise direction is the outer-bank cell. The computational results are generally good and could capture both the secondary current of the first kind and the outer-bank cell at each section. However, some discrepancies are found in characteristics of the outer-bank cell. The most conspicuous difference is the shape of the outer-bank cell. Though the horizontal length of the outer-bank cell is larger than the vertical one in the experimental results at $90^{\circ}$ and $120^{\circ}$ sections, the shape of the cell in the computational results is almost circular.

\subsection{Comparison of velocity and Reynolds stress profiles}

Figure 4 (a) and (b) show the comparison of velocity profiles and Reynolds stress profiles at the cross-section of center angle $=120^{\circ}$. W shows the width of the channel. The profiles along 5 vertical lines $0.3 \mathrm{~W}, 0.5 \mathrm{~W}, 0.7 \mathrm{~W}, 0.9 \mathrm{~W}$ and $0.95 \mathrm{~W}$ apart from the inner-bank are shown. In those figures, the computational results by LES performed by Wim van Balen et al (2008) are shown together. The present computational results agree well with the experimental result except the profiles of vertical velocity $\mathrm{W}$ at $0.9 \mathrm{~W}$ and $0.95 \mathrm{~W}$. In the experimental result of $\mathrm{U}$ at $0.95 \mathrm{~W}$, the maximum velocity appears not at the surface but around $\mathrm{z} / \mathrm{H}=0.5$. This inverse velocity profile near the surface is a driving force of the outer-bank cell. The present RANS computation over-predicted this inverse gradient and the LES result under-predicted it. The present computation also captured general aspects of Reynolds stress profiles satisfactorily. Those results indicated that the present RANS computations are competitive with the LES computations except uv profiles at $0.3 \mathrm{~W}$ and $0.5 \mathrm{~W}$, where LES results fit much better than the RANS results. Considering the computational efficiency, the present non-linear RANS model seems to be a practical tool for predicting flow structures around a sharp bend. 


\section{CONCLUSIONS}

We reported a 3D computational result of open channel flow with a sharp bend using a numerical method based on a non-linear RANS model. The present model could capture well the generation of the secondary current of the first kind as well as the outer-bank cell. The profiles of velocity and Reynolds stress profiles agree satisfactorily with the experimental result by Blanckaert et al (2002). Those results indicated the present computational model is a practical tool for predicting open channel flows with a sharply curved region.

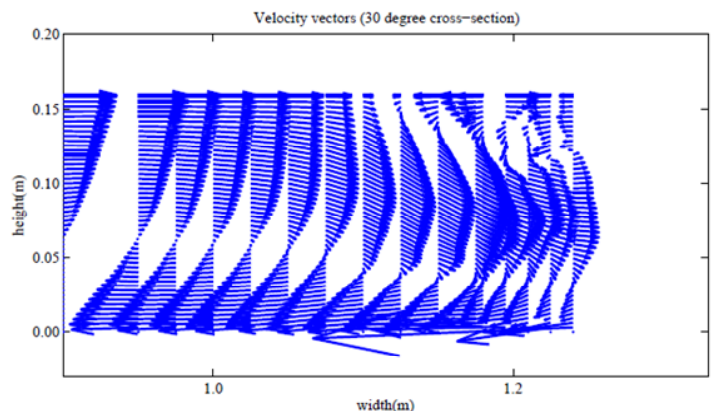

(1) $30^{\circ}$

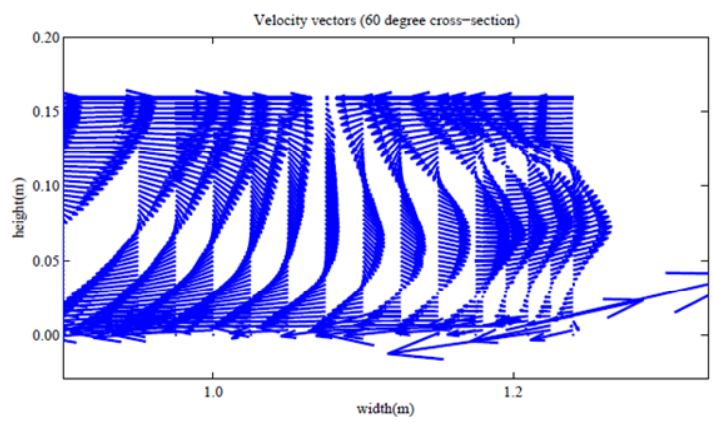

(2) $60^{\circ}$

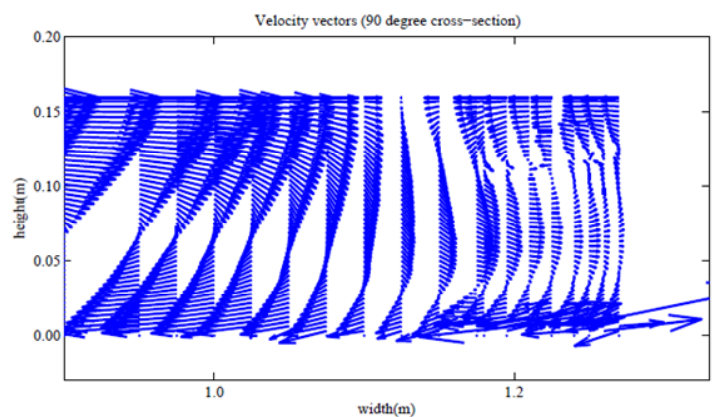

(3) $90^{\circ}$

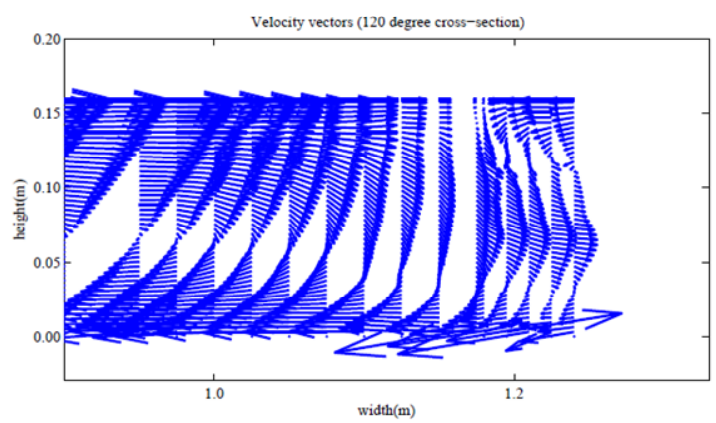

(4) $120^{\circ}$

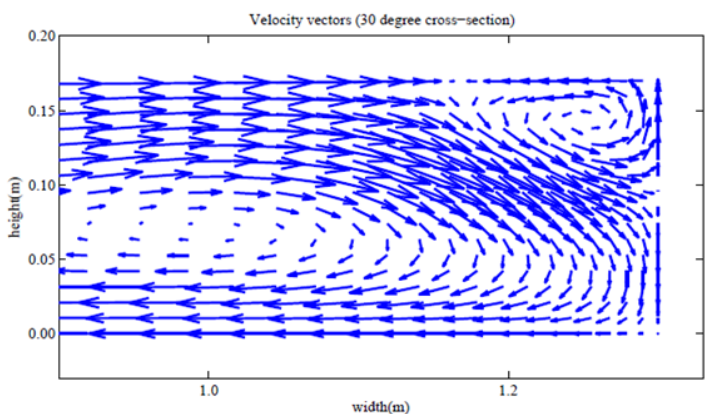

(1) $30^{\circ}$

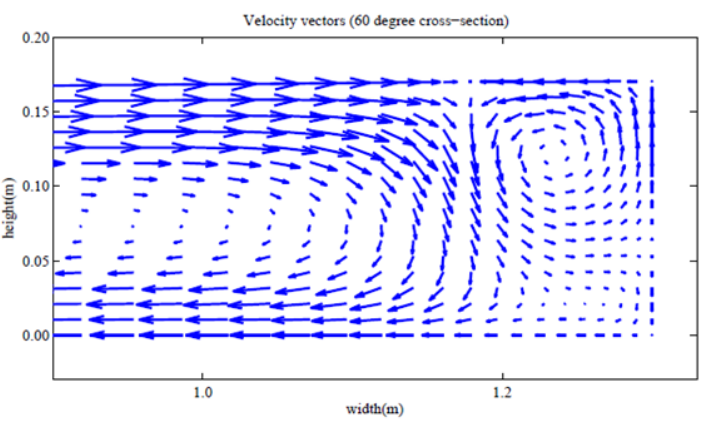

(2) $60^{\circ}$

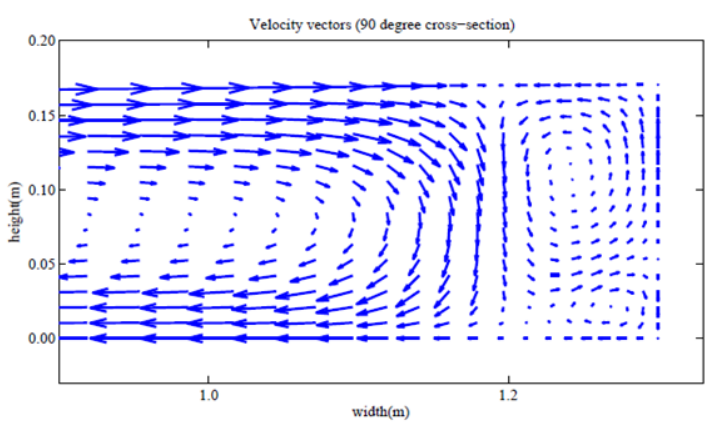

(3) $90^{\circ}$

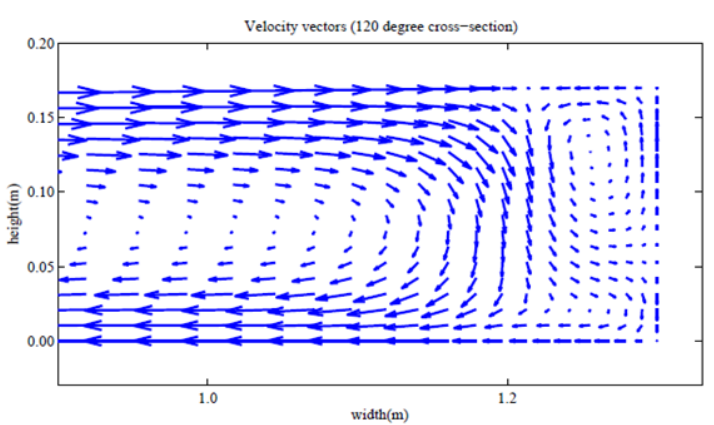

(4) $120^{\circ}$

Figure 3. Comparison of cross-sectional flow patterns (left:experiment, right:computation). 

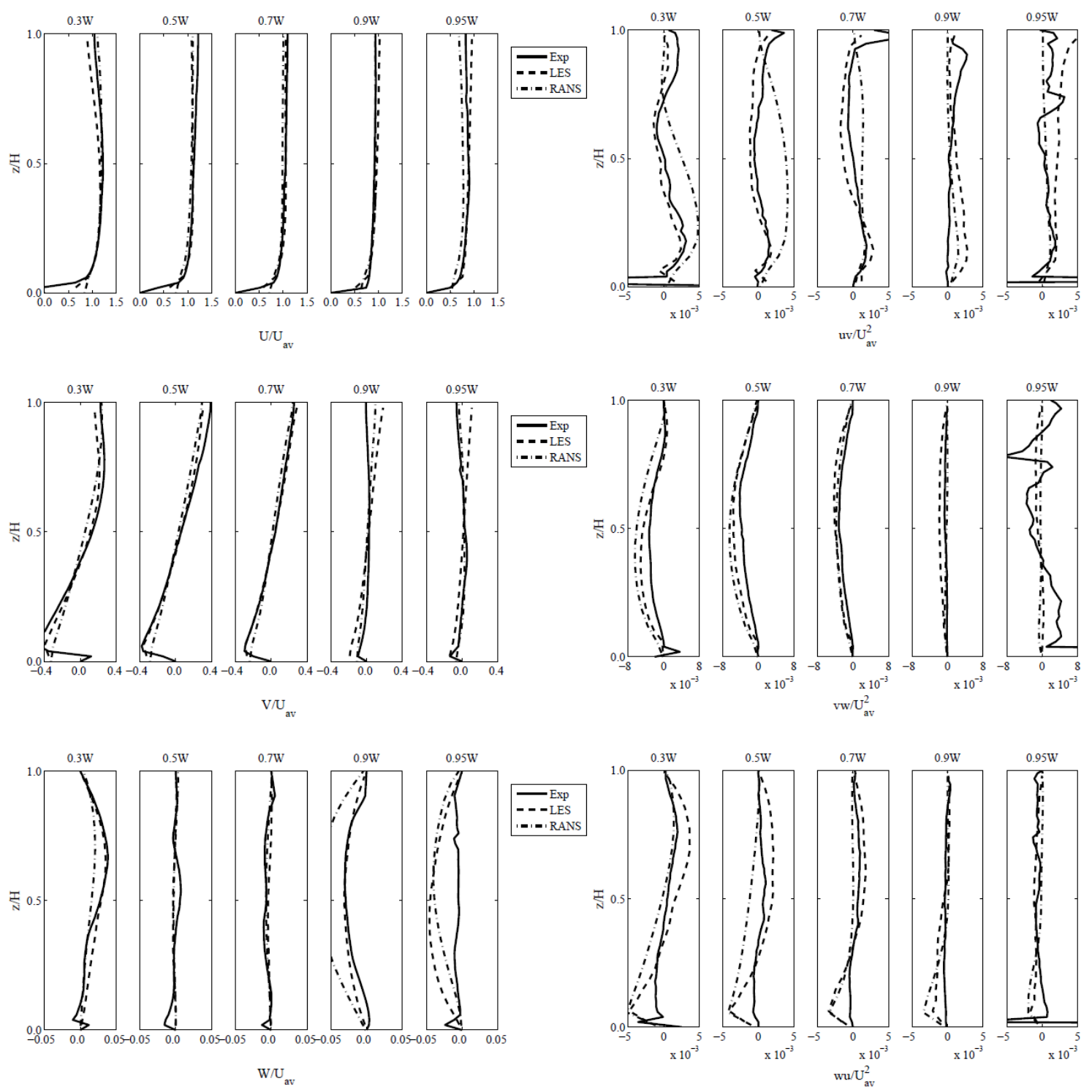

(a) Mean velocity profiles

(b) Reynolds stress profiles

Figure 4. Comparison of velocity and Reynolds stresses along several vertical sections.

\section{ACKNOWLEDGEMENTS}

Authors thank Prof. Wim S. J. Uijttewaal and Wim van Balen at Delft University of technology for provision of their LES computational data.

\section{REFERENCES}

Balen, W, Uijttewaal, W.S.J. and Blanckaert, K : LES and RANS computations of schematizes river bends, Proc. River Flow 2008, 1, pp109-117, 2008.

Blanckaert, K. (2002). Flow and turbulence in sharp open-channel bends, PhD thesis 2545, Ecole Polytechnique Federale.

Ali, M. S., Hosoda, T. and Kimura, I. (2007). A non-linear k- $\varepsilon$ model to predict the spatial change of turbulent structures in large scale vortices, J. of Applied Mech., JSCE, 10, 723-732.

Kimura, I., Uijttewaal, W.S.J., Balen, W. (2008). Application of the non-linear k- $\varepsilon$ model for simulating curved open channel flows, Proc. River Flow 2008, 1, 99-108. 